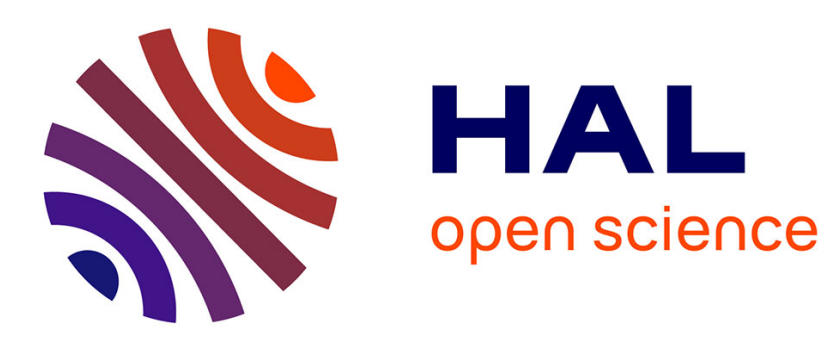

\title{
Créer de la valeur par le crowdsourcing: la dyade Innovation-Authenticité
}

\author{
Jean-Fabrice Lebraty, Katia Lobre
}

\section{To cite this version:}

Jean-Fabrice Lebraty, Katia Lobre. Créer de la valeur par le crowdsourcing: la dyade InnovationAuthenticité. Systèmes d'Information et Management, 2010, 15 (3), pp.9-40. halshs-00545780

\section{HAL Id: halshs-00545780 \\ https://shs.hal.science/halshs-00545780}

Submitted on 12 Dec 2010

HAL is a multi-disciplinary open access archive for the deposit and dissemination of scientific research documents, whether they are published or not. The documents may come from teaching and research institutions in France or abroad, or from public or private research centers.
L'archive ouverte pluridisciplinaire $\mathbf{H A L}$, est destinée au dépôt et à la diffusion de documents scientifiques de niveau recherche, publiés ou non, émanant des établissements d'enseignement et de recherche français ou étrangers, des laboratoires publics ou privés. 


\title{
Créer de la valeur par le crowdsourcing : la dyade Innovation-Authenticité
}

Jean-Fabrice Lebraty - Katia Lobre

Laboratoire GREDEG, UMR 6227 CNRS - Université de Nice Sophia-Antipolis.

Résumé

Cet article concerne une nouvelle modalité d'externalisation: le crowdsourcing. Il vise à répondre à la question suivante : Comment une opération de crowdsourcing permet-elle de créer de la valeur pour une organisation ? Dans le cadre d'une approche interprétative, une étude exploratoire empirique a été réalisée. Elle montre l'existence d'un lien entre le type de foule, ses motivations et les formes que la valeur peut prendre, à savoir : l'innovation ou l'authenticité. La mise en évidence de ce lien constitue l'un des principaux apports théoriques de cette recherche. Par ailleurs, les réponses apportées à la problématique choisie induisent les fondements d'un Business Model destiné aux entreprises qui gèrent des opérations de crowdsourcing. Ce Business Model a pour objet d'aider les organisations à capter la valeur potentiellement créée par la foule dans leurs opérations de crowdsourcing.

Mots-Clés : Crowdsourcing - Valeur - Foule - Innovation - Authenticité - Motivation

\begin{abstract}
This article is focused on a new way of externalization named : crowdsourcing. It aims to answer to the following research question: how a crowdsourcing operation can lead to value creation for an organization. From an interpretive point of view and based upon a vast empirical exploratory study, we display the link between crowd types and forms of value (authenticity or innovation). Our answer lays the foundation of a business model dedicated to crowdsourcing.
\end{abstract}

Key Words: Crowdsourcing - Value - Crowd - Innovation - Authenticity - Motivation 


\section{Créer de la valeur par le crowdsourcing : la dyade Innovation-Authenticité}

\section{Introduction}

$1^{\text {er }}$ juillet 2010, le Royaume Unis pris dans la tourmente des déficits a besoin de solutions inédites pour tenter de surmonter cette périlleuse épreuve. Le vice-Premier Ministre Nick Clegg, à l'affût de toute nouvelle opportunité, lance un site web qu'il décrit de la manière suivante "This is the most ambitious online crowdsourcing exercise ever attempted by any British government. It is an entirely new way for government to engage with people. ${ }^{1} \gg$. En quatre ans seulement, on se trouve déjà loin de l'émergence du concept de crowdsourcing [CS] qui se situe dans un article de juin 2006 (Howe, 2006) du magazine Wired. Lebraty (2009) le définit de la manière suivante : "le crowdsourcing signifie l'externalisation par une organisation, via un site web, d'une activité auprès d'un grand nombre d'individus dont l'identité est le plus souvent anonyme ». Selon cette définition, c'est le recours à une foule anonyme plutôt qu'à un fournisseur préalablement sélectionné qui différencie le plus fondamentalement le crowdsourcing de l'outsourcing. D'ailleurs Lebraty propose l'expression «externalisation ouverte» pour traduire le nouveau concept, et cela dans une double intention :

- différencier le CS de l'externalisation "classique " auprès d'une organisation préalablement identifiée et sélectionnée correspondant à une « externalisation fermée ».

- exprimer l'esprit fondant ce concept, esprit se rapprochant de celui qui prévaut dans le monde du logiciel « open-source » (Gosain, 2003);

CS et open source ne doivent toutefois pas être confondus. Si l'ouverture à la foule est commune aux 2 approches, le CS se différencie de l'open source par le fait qu'il est nécessairement initié par une organisation qui en attend des bénéfices qui lui seront exclusifs. Il se distingue également par l'absence de collaboration entre les individus dans la foule. Tous les recours à la foule via le web ne constituent donc pas nécessairement des opérations de CS. De la même manière le CS n'est qu'une des modalités possibles de l'open innovation qui repose sur l'idée que dans un monde de connaissance distribuée, les organisations ne peuvent plus compter uniquement sur leur propre R\&D. Il est plus intéressant pour elles d'externaliser en partie ces fonctions, de manière ouverte aussi bien que fermée, mais également de valoriser les inventions non utilisées en interne (Chesbrough et Appleyard, 2007, Chesbrough, 2006a). C'est ainsi que Chesbrough distingue 2 types d'innovations : les «outside-in » et «insideout »; les outside-in consistent pour une organisation à utiliser des idées ou des technologies extérieures pour son développement interne, ce qui est le cas du CS (Chesbrough, 2006b).

A l'origine, J. Howe avait défini quatre grands types de CS en fonction du type d'activité externalisé. Le tableau suivant reprend ces quatre catégories :

\begin{tabular}{|l|l|l|}
\hline Types de CS & Types d'activités & Exemples \\
\hline Crowdfunding & Financement de projet & Ulule \\
\hline Crowdcreation & Génération de nouvelles idées & PlanetInnov \\
\hline Crowdvoting & Avis sur un choix futur & Newsfutures \\
\hline Crowdwisdom & Faire appel à la sagesse populaire & Your Freedom \\
\hline
\end{tabular}

Tableau 1 : Types de crowdsourcing et types d'activités

${ }^{1}$ http://news.bbc.co.uk/2/hi/uk_news/magazine/8788780.stm 
Cependant, quatre années plus tard, le concept s'est largement développé ${ }^{2}$, le nombre d'activités ainsi externalisées s'est fortement accru, tout comme les nouvelles manières de segmenter ce concept. Guittard et Schenk (2010) par exemple, distinguent deux types de CS :

- Un CS intégratif qui a pour enjeu la mise en commun d'éléments complémentaires apportés par des individus au sein de la foule. Il concerne des tâches pour lesquelles la valeur ajoutée du CS ne provient pas des capacités individuelles des participants dont les compétences et le degré d'implication sont minimes, mais de l'addition de tâches simples et très nombreuses qui supposent d'importantes ressources.

- Un CS sélectif où la firme cliente va chercher à sélectionner une production provenant de la foule parmi un portefeuille de choix que celle-ci aura fourni. Il concerne des tâches complexes et/ou créatives pour lesquelles la valeur ajoutée du CS provient de l'originalité, de la singularité des contributions de la foule qui seront mises en concurrence par l'organisation demandeuse.

Nous avons délibérément écarté le CS intégratif portant sur des tâches routinières dans le cadre de cet article car, de manière très classique en matière d'externalisation, il est exclusivement motivé par la recherche d'une réduction de coûts, tandis que le CS sélectif de tâches complexes et/ou créatives, outre la réduction des coûts relèvent d'autres attentes de la part de leurs commanditaires ${ }^{3}$.

Ainsi, la question générale guidant cette recherche peut s'exprimer de la manière suivante :

\section{Comment une opération de crowdsourcing permet-elle de créer de la valeur pour une} organisation ?

Nous focaliser sur le «comment » suppose résolue la question de savoir si le CS permet de créer de la valeur. Or, dans tous nos entretiens avec des acteurs du CS, il est apparu évident que ce dernier y parvenait, la croissance rapide de ces acteurs en témoigne d'ailleurs. La question du «comment» s'avère nettement plus délicate et riche d'enseignements. Aussi, nous l'aborderons à travers une revue de littérature en explicitant les moteurs qui conduisent une foule à créer ce dont profite le CS. Cette potentialité créatrice n'est pas spécifique au CS, mais caractérise le fonctionnement de la foule ; on la retrouve d'ailleurs dans les concepts d'open innovation et d'open source. Dans le cas du CS, l'utilisation du media Internet contribue en sus, comme nous le verrons, à réduire les coûts et favorise la démultiplication des opportunités de solutions adaptées pour les organisations qui le pratiquent.

A la problématique retenue est attaché un double enjeu :

- Le premier est d'ordre managérial. Il consiste à contribuer à la réussite des opérations de $\mathrm{CS}$, de plus en plus nombreuses et portant sur des activités extrêmement variées. De manière plus précise, il s'agit d'aider les organisations gérant des opérations de CS, à bâtir un business model leur permettant de capter la valeur potentiellement créée par la foule et ainsi de les épauler dans leur démarche stratégique. Indirectement les organisations recourant au CS par le biais d'un prestataire bénéficieront également de la formalisation proposée.

- Le second est d'ordre théorique et consiste à proposer des pistes pour construire un modèle théorique s'appliquant pleinement au CS. En effet, les approches traditionnelles utilisées pour décrire les phénomènes d'externalisation (théorie des coûts de transaction ou approche fondée sur les ressources) ne permettent de rendre compte que partiellement des apports et conséquences du recours à cette nouvelle modalité.

Afin de traiter cette problématique, nous expliciterons dans un premier temps la méthodologie de recherche retenue et les concepts théoriques supports de ce travail. Dans un second temps

\footnotetext{
${ }^{2}$ A titre d'illustration, une recherche dans Google sur le mot crowdsourcing affichait 34000 liens fin 2006, elle aboutit aujourd'hui à près de 8 millions de liens.

${ }^{3}$ Dans la suite de cet article le terme de CS sera donc systématiquement entendu comme CS sélectif.
} 
nous exposerons nos résultats en répondant au préalable aux deux sous-questions suivantes, apparues dans la cadre la démarche interprétative retenue :

- Comment appréhender la foule pour capter sa valeur potentielle?

- Quels rapports s'établissent entre types de foule et forme de valeur?

A partir des réponses à ces questions, nous proposerons un business model favorisant la captation de la valeur potentiellement créée par la foule dans le cadre d'une opération de CS. Enfin nous discuterons ces résultats à la lumière d'exemples typiques d'opérations de CS.

\section{Design de la recherche et gestion des données}

Cet article se fonde sur plus de trois années d'investigation dans le crowdsourcing; nous avons été amenés à rencontrer près de 65 acteurs impliqués dans cette modalité non seulement en France, mais également dans plusieurs pays du moyen orient, et en Chine. A ce titre, cette recherche constitue une étude exploratoire empirique couvrant un large champ, inédite sur ce thème. Nous spécifierons dans un premier point les fondements méthodologique de la recherche en insistant sur la position épistémologique retenue et sur la méthode spécifiquement employée. Dans un deuxième point, nous détaillerons la manière avec laquelle les données de terrain ont été gérées, en précisant leur mode de collecte et les techniques d'analyse utilisées.

\subsection{Méthodologie}

Nous exposerons ici l'influence exercée par notre positionnement épistémologique sur nos choix méthodologiques.

\subsubsection{Position épistémologique}

Etudier un concept récent, issu de l'observation des comportements dans leur contexte, nous paraît compatible avec une posture épistémologique inspirée de l'interprétativisme, souvent adoptée en système d'information (Klein et Myers, 1999). Dans ce cadre, nous admettrons que le sens accordé à un concept ou à un évènement est aussi important que l'évènement luimême. Le tableau suivant récapitule les sept principes que nous avons retenus pour une recherche interprétative (Moe et al., 2010) et les mises en œuvre que nous avons pu opérer sur ces bases :

\begin{tabular}{|l|l|}
\hline \multicolumn{1}{|c|}{ Principes retenus } & \multicolumn{1}{|c|}{ Mise en ouvre } \\
\hline Principe du cercle herméneutique & $\begin{array}{l}\text { Compréhension de l'opérationalisation d'une } \\
\text { opération de CS, et proposition de concepts } \\
\text { (valeur et foule) examinés sous différents angles. }\end{array}$ \\
$\begin{array}{l}\text { A travers les discussions avec des acteurs et } \\
\text { chercheurs d'horizons différents, émergence de } \\
\text { nouvelles notions (innovation, authenticité et } \\
\text { motivation) renforçant la compréhension des } \\
\text { modes de fonctionnement du CS. }\end{array}$ \\
\hline $\begin{array}{l}\text { Principe de contextualisation } \\
\text { et sujet }\end{array}$ & $\begin{array}{l}\text { Evocation à plusieurs reprises du rôle important } \\
\text { joué par le contexte dans le développement du CS. }\end{array}$ \\
\hline $\begin{array}{l}\text { Principe d'abstraction } \\
\text { généralisation }\end{array}$ & $\begin{array}{l}\text { Conseil 2 entreprises de CS et diffusion de cette } \\
\text { modalité à une trentaine de chefs d'entreprises } \\
\text { novices souhaitant réaliser ce type d'opération. } \\
\text { Cela a rendu possible la signature de 2 contrats de } \\
\text { CS. }\end{array}$ \\
\hline Principe de raisonnement dialogique
\end{tabular}




\begin{tabular}{|l|l|}
\hline & $\begin{array}{l}\text { les modèles théoriques et la mise en œuvre du CS } \\
\text { telle que vécue par les acteurs. }\end{array}$ \\
\hline Principe des interactions multiples & $\begin{array}{l}\text { Pour obtenir de nombreuses et potentiellement } \\
\text { contradictoires interprétations de nos cas, prise de } \\
\text { contacts avec l'ensemble des acteurs et des } \\
\text { sources d'informations disponibles. }\end{array}$ \\
\hline Principe du soupçon & $\begin{array}{l}\text { Recherche constante des causes profondes des } \\
\text { réponses des acteurs et du sens caché de ces } \\
\text { dernières. C'est ainsi par exemple qu'a pu émerger } \\
\text { la notion de «création authentique ». }\end{array}$ \\
\hline
\end{tabular}

Tableau 2 : mise en œuvre des 7 principes d'une recherche interprétative.

\subsubsection{Choix méthodologiques}

L'approche adoptée est principalement qualitative. Nous nous attachons à comprendre le sens donné par les acteurs aux évènements dans leurs contextes.

Cette approche est pertinente dans l'investigation d'un phénomène nouveau et dynamique. Nouveau parce que le CS est apparu en juin 2006. Dynamique par l'évolution permanente du concept même de CS. Nous sommes en effet, encore en phase de foisonnement conceptuel, phase au cours de laquelle le terme de CS est employé à propos d'activités fort différentes et en constant développement.

Nous nous sommes fondés sur une étude de cas multiples afin de mieux cerner les liens entre un phénomène (le crowdsourcing) et les perceptions de sa valeur par les chefs d'entreprises.

\subsection{Gestion des données}

Nous avons majoritairement manipulé des données qualitatives et quelques données quantitatives issues de deux questionnaires comme nous allons l'expliciter.

\subsubsection{Mode de collecte des données}

Nous avons recueillis les données selon trois modalités différentes, données qui peuvent être ensuite rangées en deux catégories, primaires ou secondaires.

La première des modalités a débuté en 2006 et correspond à une phase de découverte du concept de CS. A ce moment-là, il n'y avait que peu d'acteurs avisés en France sur ce thème, aussi, nous nous sommes appuyés sur le blog mis en ligne par J. Howe ${ }^{4}$. Une fois le concept cerné, nous avons continué à effectuer une veille sur ce blog, puis nous avons pris en compte un second site qui nous apparaissait pertinent ${ }^{5}$. Parallèlement au suivi des blogs nous avons collecté l'ensemble des articles académiques ou professionnels traitant du CS.

La seconde modalité de collecte des données a débuté en janvier 2008 et a consisté en la recherche d'acteurs impliqués dans le CS ou susceptibles de l'être. Ainsi, nous avons organisé des rencontres entre acteurs et entrepreneurs intéressés. Nous avons pris des contacts avec 5 entreprises réalisant du CS et 5 autres l'utilisant. En outre, nous avons explicité le concept de CS devant des étudiants, cadres ou dirigeants d'entreprises, ce qui nous a permis de recueillir de nombreuses opinions. Toutes ces rencontres et contacts ont débouché sur 72 entretiens, de

\footnotetext{
${ }^{4}$ Intitulé «crowdsourcing »- voir Webographie. Nous avons aspiré l'ensemble du contenu du blog au sein duquel nous avons retenu 103 commentaires liés à des billets postés par le concepteur du site, nous apparaissant pertinents. Ces commentaires provenaient en majorité d'utilisateurs identifiables (59\%). Cette collecte s'est déroulée du $1^{\text {er }}$ janvier 2007 au 15 juin 2010

${ }^{5}$ Crowdsourcingresults - voir Webographie. Seul 5 commentaires ont retenu notre attention à ce jour.
} 
type semi-directif ou non directifs d'une durée comprise entre $1 \mathrm{~h}$ et $3 \mathrm{~h}$. Ces entretiens réalisés en face à face et/ou par téléphone se sont déroulés entre janvier 2008 et juin $2010^{6}$.

Enfin, la dernière modalité est plus récente. Débuté en, 2010, elle consiste en une activité de conseil auprès d'entreprises dont le CS constitue le cœur de métier, afin d'épauler leurs dirigeants dans la réalisation de leur Business Model. Cette activité permet d'assurer une validité externe aux résultats et de collecter de nouvelles informations permettant d'affiner ces derniers (Modell, 2005).

Décrivons à présent, selon le critère du type de données (primaires ou secondaires), les éléments recueillis.

\begin{tabular}{|c|c|}
\hline Données primaires & Données secondaires \\
\hline $\begin{array}{l}\text { - } 2 \text { Blogs consacrés au CS. } \\
72 \text { entretiens auprès d'acteurs du CS } \\
\text { (décisionnaires d'entreprises ayant, } \\
\text { soit recours au CS, soit souhaitant y } \\
\text { recourir ou encore souhaitant } \\
1 \text { 'étudier); } \\
\text { - } 2 \text { questionnaires ( } 1 \text { adressé à une } \\
\text { communauté d'internautes } \\
\text { participants à des opérations de CS } \\
\text { et } 1 \text { adressé à un groupe de chefs } \\
\text { d'entreprises ayant participé à un } \\
\text { séminaire dédié au CS) }\end{array}$ & $\begin{array}{l}\text { - Les documents disponibles issus de } \\
63 \text { entreprises pratiquant le CS (aux } \\
\text { USA et en France). } \\
\text { - Articles centrés sur le concept de CS } \\
\text { et répartis de la manière suivante : } \\
12 \text { articles et communications } \\
\text { académiques et } 27 \text { articles dans des } \\
\text { revues professionnelles. }\end{array}$ \\
\hline
\end{tabular}

Tableau 3 : les types de données recueillies

\subsubsection{Méthode d'analyse des données}

Est concernée ici l'analyse des données primaires collectées (blogs, entretiens et questionnaires). Les données secondaires ont été traitées afin de réaliser un fond documentaire avec un double objectif : comparer nos travaux avec ceux d'autres chercheurs, et opérer une veille continue sur les modèles d'affaires des entreprises de CS, afin de vérifier si nos résultats restaient cohérents avec la réalité des entreprises.

Concernant les blogs, ils ont fait l'objet d'une analyse qualitative de contenu. Plus précisément, ce sont les commentaires postés sur les 2 blogs énoncés plus haut qui ont été analysés. Nous avons choisi de coder l'ensemble des données, afin d'avoir une vision globale par thème du phénomène de crowdsourcing, tel que les acteurs l'expriment (Thiétard, 1999: 460). Pour ce faire, nous avons adopté le cheminement suivant :

- lecture des commentaires par référence à la problématique

- formulation des objectifs de l'analyse : rechercher les éléments relatifs au concept de valeur, les éléments concernant la foule dans le CS, les impacts possibles du CS et l'ampleur estimée de ce phénomène ;

- mise en place d'un codage ouvert (description des idées à repérer et des mots les constituant) ;

- classification en thèmes ;

- analyse par comptage des mots et des thèmes ;

- formalisation des interprétations.

Les résultats du codage peuvent être succinctement présentés en spécifiant les thèmes et mots retenus.

\begin{tabular}{|l|l|}
\hline Thèmes & Idées - Mots \\
\hline
\end{tabular}

\footnotetext{
${ }^{6}$ Ajoutons que nous poursuivons ces actions afin d'accroître notre stock d'entretiens et d'observations et de compléter nos travaux sur le sujet.
} 


\begin{tabular}{|l|l|}
\hline Ampleur du CS & $\begin{array}{l}\text { Stratégique (important }- \text { pertinent }- \text { stratégique - } \\
\text { déterminant) } \\
\text { Temporaire (mode, test, initiative) }\end{array}$ \\
\hline Spectre d'activités & $\begin{array}{l}\text { R\&D (R\&D, idées, pistes, produit, service, procédé) } \\
\text { Commercial (vente, profit) } \\
\text { Communication (idées, campagnes, créer, diffuser, } \\
\text { contrôler) } \\
\text { Production (co-production, produit, service) }\end{array}$ \\
\hline Valeur & $\begin{array}{l}\text { Coût (coût, prix, diminution) } \\
\text { Démultiplication des opportunités } \\
\text { Revenus } \\
\text { Création (innovation, authenticité) } \\
\text { Terme (durée, long terme, court terme, temporalité, rapidité, } \\
\text { facilité) }\end{array}$ \\
\hline Motivations & Intrinsèques \\
Extrinsèques \\
Extrinsèques internalisées
\end{tabular}

Tableau 4 : Codage

Concernant les entretiens, nous avons défini trois catégories d'entreprises ayant recours au CS :

\begin{tabular}{|c|c|c|}
\hline Catégorie 1 & Catégorie 2 & Catégorie 3 \\
\hline $\begin{array}{l}\text { Les entreprises qui } \\
\text { gèrent elles-mêmes leur } \\
\text { activité de CS sur leur } \\
\text { propre site web ou sur un } \\
\text { site qu'elles ont créé à } \\
\text { cet effet. }\end{array}$ & $\begin{array}{l}\text { Les entreprises qui ont } \\
\text { fait du CS leur cour de } \\
\text { métier. Elles gèrent donc } \\
\text { des activités de CS pour } \\
\text { le compte d'entreprises } \\
\text { clientes dans le cadre de } \\
\text { relations en B to B. }\end{array}$ & $\begin{array}{l}\text { Les entreprises qui externalisent } \\
\text { leurs activités de CS auprès d'un } \\
\text { intermédiaire spécialisé dans cette } \\
\text { activité (entreprises de la } \\
\text { catégorie 2) }\end{array}$ \\
\hline
\end{tabular}

Tableau 5 : catégories d'entreprises pratiquant le CS

Nous avons mené des entretiens auprès de dirigeants d'entreprises appartenant à chacune des trois catégories précitées. Examinons, la manière avec laquelle nous avons traité les entretiens réalisés.

La nouveauté du phénomène, en particulier en France, limite de fait le nombre des interviewés potentiels. Cependant, 2 dirigeants d'entreprises de la catégorie 1, 3 dirigeants de la catégorie 2 et 5 dirigeants de la catégorie 3 ont été interrogés dans le cadre d'entretiens semi-structurés autours des thèmes et idées mis à jour dans l'analyse documentaire. Le guide d'entretien était donc structuré comme suit :

1. Recours au CS (Quand ? Comment ? Dans quel cadre/contexte ? Avec qui ? Pourquoi ?)

2. Réaction initiale face au CS (Favorable/défavorable ? Menace/opportunité)

3. Caractéristiques des contributeurs et de leurs contributions aux opérations de CS.

4. Conséquences du recours au CS (En termes de métier/d'activité/d'organisation/de partenaires ? En termes de coûts/profits/qualité/délais ?)

5. Difficultés/limites/dangers autour du CS ?

Le caractère stratégique du CS justifie de s'adresser aux décideurs. A cet effet, nous avons mené des entretiens semi-structurés et non structurés. Ces entretiens répondent bien aux objectifs de vérification et d'approfondissement des idées et réflexions de l'analyse documentaire qui leurs sont assignés (Blanchet et Gotman, 1992). Les entretiens ont été intégralement retranscrits. Parallèlement, ils ont fait l'objet d'une prise de notes sous la forme 
de fiche de synthèse d'entretien dont l'objet est de mettre en évidence les éléments forts du discours de l'interlocuteur. Le logiciel Nvivo8 a été utilisé pour indexer les propos des interlocuteurs interrogés sur la base des thèmes initiaux et les enrichir de nouveaux thèmes à partir du contenu des entrevues.

Dans une logique de validation externe, les résultats de nos investigations ont été présenté lors de deux séminaires « recherche / entreprise » autour du CS. Participaient notamment à ces séminaire, $24+27$ dirigeants d'entreprises aguerries aux nouvelles technologies, mais sans toutefois recourir au CS et parfois même en en méconnaissant la pratique ${ }^{7}$.

Enfin les deux questionnaires ont fait l'objet de traitements statistiques basiques. Des tableaux croisés dynamiques ont été réalisés avec Excel 2007, de manière à faire émerger des corrélations entre items, en particulier entre les caractéristiques des membres de la communauté interviewée et les types stimuli qu'ils privilégient dans leur participation à une opération de CS. Le questionnaire destiné aux chefs d'entreprise nous a surtout permis de cibler les personnes que nous avons ensuite interviewées de manière à traiter différemment leurs réponses selon qu'ils s'estimaient ou non spécialiste en CS.

Le tableau suivant résume les données collectées et les méthodes d'analyse mises en œuvre :

\begin{tabular}{|c|c|c|c|c|c|c|c|c|c|}
\hline $\begin{array}{l}\text { Types de } \\
\text { données }\end{array}$ & Blogs & \multicolumn{3}{|c|}{$\begin{array}{l}\text { Entretiens semi- } \\
\text { structurés }\end{array}$} & \multicolumn{2}{|c|}{ Entretiens non directifs } & Questionnaires & $\begin{array}{c}\text { Documents } \\
\text { d'entreprises de CS }\end{array}$ & Articles \\
\hline $\begin{array}{l}\text { Types de } \\
\text { sources }\end{array}$ & & $\mathrm{C} 1$ & $\mathrm{C} 2$ & $\mathrm{C} 3$ & $\begin{array}{c}\text { Acteurs } \\
\text { potentiels }\end{array}$ & Chercheurs & & & \\
\hline $\begin{array}{c}\text { Nombre } \\
\text { de sources }\end{array}$ & 2 & 2 & 3 & 5 & 45 & 9 & 2 & 63 & 39 \\
\hline $\begin{array}{c}\text { Volume } \\
\text { des } \\
\text { données }\end{array}$ & 108 & 5 & 8 & 5 & 45 & 9 & 2 & 63 & 39 \\
\hline Analyse & \multicolumn{6}{|c|}{ Contenu } & $\begin{array}{l}\text { Statistiques } \\
\text { simples }\end{array}$ & Confirmatoires & $\begin{array}{c}\text { Base } \\
\text { documentaires }\end{array}$ \\
\hline
\end{tabular}

Tableau 6 : synthèse de la gestion des données

En conclusion de ce point, deux caractéristiques de notre démarche sont à souligner :

- Le paradigme interprétativiste retenu, qui nous a conduits à essayer de comprendre le sens accordé par les acteurs aux concepts, notions et idées qu'ils évoquent. Cette orientation implique notamment de ne pas s'arrêter aux termes employés dans leur acception première, (valeur, innovation ou authenticité par exemple) mais de rechercher le sens que les acteurs attribuent à ces termes en accord avec les diverses acceptions qu'ils recouvrent ;

- La richesse des données collectées, tant en termes de volume que de diversité, ce qui nous a amenés à employer des méthodes d'analyse variées et adaptées.

\section{Cadre théorique}

Au regard du rôle central de la foule dans la définition du CS et de la question générale traitée, centrée sur la création de valeur, les concepts de foule et de valeur se devaient d'être examinés. Par ailleurs, conformément à l'approche interprétative retenue, nos investigations nous ont conduits à nous intéresser à 3 autres concepts : l'innovation, l'authenticité et la motivation.

\subsection{Concepts initiaux}

\subsubsection{La foule et ses mécanismes}

Le concept de foule est étudié depuis longtemps. Dans son ouvrage maintes fois ré-édité, G. Le Bon (2003) indique : «le mot foule représente une réunion d'individus quelconques...

\footnotetext{
${ }^{7}$ Séminaire au laboratoire de recherche GREDEG UMR CNRS UNS 6227 en octobre 2009 et séminaire à la Business School SKEMA - Sophia Antipolis en Mai 2010.
} 
quels que soient aussi les hasards qui les rassemblent. »(p. 2). La définition semble large, cependant il ajoute immédiatement « au point de vue psychologique, l'expression foule prend une signification toute autre. Dans certaines circonstances données et seulement dans ces circonstances, une agglomération d'hommes possède des caractères nouveaux fort différents de ceux de chaque individu qui la compose. " Il évoque alors l'idée de "foule organisée ». Nous sommes alors face à un jeu à somme variable. Parfois la somme peut être négative dans le cas où le comportement de la foule s'adosse à des valeurs considérées défavorablement comme la lâcheté ou la violence. Parfois la somme est positive et c'est le cas qui nous intéresse ici. Dans son ouvrage, J. Surowiecki (2005) stipule que deux éléments sont requis pour la constitution d'une foule sage. Le premier élément est la diversité des interprétations. Disposant de compétences variées, locales et/ou globales, les individus vont créer un sens variable par rapport à un même fait ou à une même question. Ce point est important, car il conduit à différentier une foule qui peut s'appeler communauté d'avec le concept de communauté de pratiques (Lefebvre et al., 2004). En effet, la communauté de pratique implique une certaine homogénéité des façons de faire, des attitudes et du mode de pensée, ce qui tend à réduire la diversité. Le rôle prépondérant accordé à la diversité soulève également une question d'importance : celle de la taille minimale d'une foule ? J. Howe avance le chiffre de 5000 (Howe, 2009, p. 282), mais rien n'a été scientifiquement démontré. Ce qui transparaît en revanche, est que dans une foule il existe des «cercles de qualité » qui conduisent à ce que $90 \%$ de la foule propose des idées insignifiantes, $9 \%$ des idées intéressantes, $1 \%$ des idées très intéressantes, et il est escompté qu'un $\varepsilon$ trouve l'Idée géniale.

Le deuxième élément est constitué par l'indépendance des membres de la foule. Cette indépendance se situe au niveau du traitement de l'information. L'auteur fait ici référence à la théorie de la cascade d'information (Welch, 2000). Utilisée notamment en finance pour mieux comprendre le comportement des acteurs de marché, cette approche montre le rôle prépondérant des informations externes qui conduisent à des mécanismes de mimétisme comportemental. Ce mimétisme peut aller dans un sens bénéfique (la standardisation de certains procédés) ou désastreux (les bulles financières), et peut expliquer les sommes positives ou négatives évoquées plus haut. Il en ressort que si les individus prêtent un peu moins d'attention au comportement des autres, leur performance collective semble être supérieure en moyenne (Girotra et al., 2010). Cette question des liens d'informations nous semble primordiale alors même qu'elle n'a pas été abordée par J. Howe lorsqu'il décrit les cinq caractéristiques de la foule (Lebraty, 2009). En effet, aujourd'hui un très grand nombre d'internautes appartient à un ou plusieurs réseaux sociaux ${ }^{8}$. Il ne serait donc pas surprenant que les cascades d'information, autrement dit des phénomènes de mimétisme passent à travers ces réseaux. Ce paramètre, non suffisamment étudié à ce jour, exerce de notre point de vue une certaine influence sur les mécanismes de CS. Ces cascades sont peut être un moyen théorique d'expliquer voire même d'exploiter le concept de leader d'opinion dans une foule. En effet, même si les voies de recherches sont encore largement inexplorées, attirer des leaders d'opinion correspondant à ses attentes, pourrait pour une organisation, dans le cadre d'une opération de CS, orienter de manière positive ces phénomènes de mimétismes potentiels. En l'absence de cascades, ces leaders seraient «noyés » dans la foule, en revanche en présence de cascades ils pourraient œuvrer au service de l'organisation.

Concernant une opération de CS, une foule possède deux fonctions essentielles qui peuvent être combinées : la génération d'idées et la sélection d'idées conduisant à faire émerger une solution. Il est alors possible d'utiliser une foule pour :

- Générer du contenu : la foule est un immense réservoir de compétences diverses, qui dans le cadre d'une opération de CS va générer de multiples créations individuelles.

\footnotetext{
8 L'estimation actuelle est que les $2 / 3$ des internautes occidentaux participent un réseau social (www.journaldunet.com).
} 
Ce contenu peut se ranger selon deux grandes catégories. La foule peut fournir des réponses à des problèmes posés ou offrir de nouvelles idées, fournissant ainsi de nouvelles opportunités.

- Sélectionner du contenu : la foule est un puissant mécanisme de sélection. Elle agit comme un filtre ; au travers de votes, d'avis, de commentaires, une sélection s'opère. Dans le cadre d'une opération de CS donnée, la foule peut donc être utilisée pour sélectionner les créations individuelles précédemment générées et ainsi favoriser l'émergence d'une création collective.

Par sa diversité et l'indépendance de ses membres, en générant et/ou en sélectionnant du contenu, la foule permet de créer. Ainsi, s'il y a création de valeur dans le cadre d'opérations de CS, comme les acteurs du CS s'accordent à le dire, cette valeur est avant tout créée par la foule. Mais que peut créer la foule, source de valeur pour une organisation? Autrement dit, quelle forme peut prendre cette valeur?

\subsubsection{Créer et capter de la valeur 2.0}

La valeur est un concept qui, pour les organisations, englobe une large palette d'indicateurs. Chaque champ disciplinaire a eu tendance à développer à partir de besoins spécifiques, des approches et des instruments de mesure particuliers de la valeur. Le processus de création de valeur n'est pas figé lui non plus, et dépend notamment des particularités du créateur de cette valeur : individu, organisation ou encore société (Lepak et al., 2007). Ainsi, face à la nature protéiforme du concept, la valeur sera de prime abord entendue dans cet article comme une mesure d'importance accordée par les acteurs concernés, autrement dit comme une perception. Si les acteurs du CS sont convaincus de la valeur créée par les opérations de CS qu'ils mettent en œuvre, ils la perçoivent en termes de coûts/rapidité/simplicité, ainsi qu'en termes d'innovation et d'authenticité créées par la foule.

La question de la valeur dans le cadre d'opérations de CS suppose en outre d'intégrer le média internet dans la réflexion puisqu'il en est un élément obligatoire et même constitutif. Il s'agit donc plus précisément de s'intéresser au rôle du média dans la valeur créée. De nombreux travaux montrent la spécificité des relations électroniques dans la création de valeur (Aguila-Obra et al., 2007, Möller et Rajala, 2007, Kim et al., 2010). Plus avant, dans le domaine du commerce électronique, Amit et Zott (2001) estiment que la création de valeur s'articule autour de quatre dimensions interreliées: l'efficacité, la complémentarité, l'affiliation et la nouveauté. Cet article souvent mobilisé ${ }^{9}$ nous apparaît particulièrement pertinent pour comprendre le rôle du web 2.0 dans la valeur créée par une opération de CS, au sens où il fait largement échos à nos investigations, notamment pour les dimensions nouveauté et efficacité. En effet, la capacité d'interactivité du concept de Web 2.0 permet à l'internaute de co-construire du contenu. Sans cette potentialité, pas de créations de la foule accessibles aux organisations. Au plan de la nouveauté, le média a donc permis de connecter des parties qui ne l'étaient pas précédemment (les organisations et la foule) car les concours d'affiches d'antan par exemple, n'ont que peu en commun avec les opérations de CS d'affiches publicitaires, que ce soit en termes de dimension du challenge ou des caractéristiques de la foule. Par ailleurs, le web 2.0 est susceptible de rendre les créations de la foule plus efficaces que les créations internes aux organisations ou externalisées de manière traditionnelle. En effet, à l'instar des résultats de Gaspoz (2010) concernant les marchés prédictifs appliqués à l'estimation des résultats d'élections, les créations de la foule, de qualité comparable voire supérieure aux créations traditionnelles, peuvent être obtenues de manière plus simple, à un coût et dans des délais largement inférieurs. La mobilisation du modèle d'Amit et Zott permet donc de confirmer et de mieux saisir les perceptions des acteurs en

\footnotetext{
${ }^{9} 76$ fois cités dans les articles disponibles dans sciencedirect notamment.
} 
matière de création de valeur par le CS. En effet, cette création de valeur est principalement liée à deux éléments interreliés :

- La capacité créatrice de la foule, rendue accessible aux organisations par le média internet ;

- L'efficacité en termes de coûts/simplicité/rapidité, potentiellement supérieure de ces créations sur les créations traditionnelles, inhérente aux caractéristiques intrinsèques du web 2.0.

Ainsi, dans la suite de ce travail, ce ne sont pas tant les caractéristiques du web 2.0, déjà largement étudiées qui seront examinées, mais la forme prise par la valeur créée par la foule, en termes d'authenticité et d'innovation, comme le suggèrent les perceptions des acteurs du CS.

Enfin, pour clore ce point relatif à la valeur, il convient de distinguer création de valeur et capture de la valeur. Comme l'indique Priem (2007), il peut exister une confusion entre ces deux termes. Capturer de la valeur conduit à maximiser la taille de la part que l'on peut récupérer sur la valeur totale d'un élément. Créer de la valeur consiste en revanche à essayer, notamment via l'innovation, à augmenter la valeur que l'on peut avoir sur un élément (Johannessen et Olsen, 2010) ${ }^{10}$.

La foule permet de créer de la valeur en termes d'innovation et d'authenticité en générant et/ou en sélectionnant du contenu. La capturer suppose un Business Model [BM] adapté conformément à l'approche de la valeur de Chesbrough (2006b). Le BM étant entendu ici à la suite de (Osterwalder et al., 2009) comme la logique avec laquelle une organisation crée, fournit et capture de la valeur. La construction d'un BM adapté aux opérations de CS constitue précisément l'objectif de cette recherche.

\subsection{Concepts émergeants}

«La vraie valeur pour le client c'est la possibilité de créer, c'est ce qui leur permettra de survivre. »

«On veut favoriser la création d'innovation»

«On traque les sentiments authentiques, c'est ce qui nous intéresse »

«Ce n'est pas si évident d'essayer d'attirer la foule, de comprendre pourquoi certains veulent venir. On cherche à mieux cerner leurs motivations »

Ces quatre verbatim sont représentatifs de l'importance qu'attachent les acteurs aux concepts suivants : innovation, authenticité et motivation. Innovation et authenticité sont les termes employés par les acteurs du CS lorsqu'ils s'expriment sur la valeur perçue ou la valeur attendue des opérations de CS qu'ils mettent en œuvre. La motivation a quant à elle naturellement émergée de leurs questionnements concernant les moyens permettant d'inciter la foule à participer à ces mêmes opérations de CS.

\subsubsection{Innovation et authenticité}

Nos investigations ont mis en lumière que les acteurs du CS attendent de la foule des créations innovantes et/ou authentiques. L'examen de la littérature révèle de multiples significations de l'innovation et de l'authenticité. Aussi, pour chacun, nous évoquerons des définitions académiques susceptibles de s'appliquer à notre étude et compatibles avec la vision des acteurs interviewés. Ce point a donc pour objet de définir les deux concepts au plan théorique afin d'être en mesure de les identifier et au-delà de les susciter dans le cadre d'opérations de CS.

\section{Innovation}

\footnotetext{
${ }^{10}$ Par analogie avec un gâteau, créer de la valeur suppose de faire grossir ce gâteau, capter cette valeur consiste à s'en octroyer le maximum de parts.
} 
Etymologiquement, le mot innovation vient du latin novus (nouveau) qui génère le verbe innovare défini comme le "fait d'introduire dans une chose établie, quelque chose de nouveau, d'encore inconnu». Il est associé à la notion de progrès et bénéficie d'une connotation positive, valorisante en Sciences Humaines. Il se distingue de l'invention par l'idée d'une valorisation économique de cette dernière. Ainsi, lorsque les acteurs du CS expriment des attentes en matière d'innovation, créatrice de valeur pour l'organisation, c'est bien le terme consacré qu'ils utilisent.

Pour préciser ce concept aux acceptions diverses et parfois contradictoires, nous nous appuyons sur les niveaux d'analyse suivants : la nature même de l'innovation appréhendée soit en termes de résultat, soit de processus ; les principales classifications de l'innovation ; le système de référence utilisé pour apprécier la nouveauté (Mors, 2010, Ayerbe, 2006, Crossan et Apaydin, 2010).

La nature même de l'innovation appréhendée soit en termes de résultat, soit de processus. En tant que résultat, l'innovation présente nécessairement un caractère «accompli », qu'il s'agisse d'une technologie, d'un produit ou d'un procédé. En tant que processus, l'innovation est appréhendée de manière dynamique à travers ses cheminements, ses trajectoires. Une opération de CS s'inscrit donc dans le processus d'innovation d'une organisation. Son résultat est constitué par la sélection opérée soit par cette même organisation, soit par la foule, d'une des productions de cette dernière.

Les principales classifications de l'innovation. Au nombre de deux, elles distinguent une classification selon la nature intrinsèque de la nouveauté d'une classification selon l'intensité (ou le degré) qu'elle peut prendre.

- Selon la nature intrinsèque de l'innovation une nouvelle classification émerge distinguant innovation technologique versus organisationnelle et innovation de produit versus de procédé. Le plus souvent assimilée à une innovation interne, l'innovation organisationnelle porte notamment sur les structures formelles, les règles et les procédures et les systèmes de prise de décision. De ce fait elle ne concerne pas, pour l'instant, les opérations de CS. L'innovation technologique quant à elle, concerne à la fois les produits et les procédés, qu'ils soient nouveaux ou améliorés, et relèvent plutôt d'un référentiel externe (la sanction du marché) pour les premiers et interne (l'évaluation de l'organisation) pour les seconds. Remarquons que les opérations de CS portent indifféremment sur des innovations produits et procédés.

- L'intensité ou le degré de l'innovation peut être représenté sur un continuum allant du radicale à l'incrémentale. Dès lors l'innovation sera qualifiée de radicale lorsqu'elle repose sur des connaissances nouvelles, parfois totalement étrangères aux savoir-faire traditionnels. A l'autre extrémité, l'innovation sera dite incrémentale car n'impliquant pas de savoir-faire fondamentalement nouveaux et reposant sur une amélioration progressive des connaissances existantes. Les attentes en matière de créations innovantes des opérations de CS relèvent plutôt de l'innovation radicale, cependant, comme nous le verrons, le CS peut mener à des innovations incrémentales.

Le système de référence utilisé pour apprécier la nouveauté. Deux référentiels dominent la littérature en tant qu'extrémités d'un continuum :

- l'organisation ou référentiel minimal dans le cadre duquel il y a innovation lorsque l'entreprise effectue quelque chose qu'elle n'avait jamais effectué auparavant.

- Le marché ou référentiel maximal. Dans cette acception, l'innovation ne sera effective que si elle est accomplie pour la première fois dans l'absolu.

Les créations innovantes attendues des opérations de CS sont le plus souvent jugées comme telles par l'organisation qui, par référence à elle-même, apprécie la nouveauté. En effet, lorsqu'elle propose un challenge, l'organisation espère bénéficier des compétences d'individus dont le domaine d'expertise est suffisamment éloigné du sien, pour obtenir de 
solutions innovantes grâce à des processus analogiques ou des phénomènes de transposition. Ces solutions n'apparaissent pas nécessairement nouvelles pour le marché mais, elles le sont pour l'organisation.

En résumé, les attentes en termes de créations innovantes d'une opération de CS s'expriment par la nouveauté plutôt radicale, d'un produit ou d'un procédé, nouveauté établie par l'organisation et obtenue grâce à l'expertise d'individus dans la foule dont le domaine de compétence est rarement identique à celui de l'organisation.

\section{L'authenticité}

Les attentes en termes de créations authentiques des acteurs du CS sont, dans leur très grande majorité, reliées à une marque (Légo, Dell, Gervais, Kickers...). Elles orientent dès lors ce travail de définition vers l'authenticité de la marque.

Selon Kolar et Zabkar, (Kolar et Zabkar, 2010), l'authenticité de la marque peut être définie à partir de dimensions cognitive ou expérientielle. L'image cognitive permet de mettre en exergue les croyances sur lesquelles les consommateurs semblent s'appuyer pour établir l'authenticité d'une marque. Selon cette approche, une marque authentique pourrait se définir comme une marque perçue d'origine, ancrée dans une origine et donc singulière, sincère ou encore qui fait autorité, conformément à sa racine grecque authentikos ${ }^{11}$.

Si des dimensions cognitives transparaissent dans la littérature et les pratiques commerciales pour traduire l'authenticité de la marque, celle-ci émerge également d'éléments affectifs et sensoriels. Sous son angle expérientiel, la marque authentique suppose une connexion intime, voire passionnelle entre la marque et le consommateur (Gobe, 2001). La marque constitue le catalyseur d'une expérience qui transporte le consommateur dans ses souvenirs (Cova et Cova, 2001: 78) et/ou lui permet de créer ou d'affirmer son identité personnelle (MacIntosh et Prentice, 1999). La marque authentique requiert donc un lien fort, une véritable proximité entre la marque et le consommateur qui suscitera l'investissement de ce dernier dans la marque, sa participation à sa construction et à son image.

Cet effort de définition de la marque authentique éclaire les attentes des acteurs du CS en matière de créations authentiques. En effet, ils attendent de la foule des créations singulières, originales donc authentiques dans l'acception cognitive du concept. De plus, ils attendent de la foule qu'elle participe, à travers ses créations, à la construction de l'image et l'identité de la marque, qui seront d'autant plus authentiques que la proximité de la foule avec la marque sera importante. Cette nécessaire proximité conduit ici à réduire la foule aux consommateurs de la marque, avec pour conséquence la transformation de la foule en une communauté-tribu.

Afin de développer et de nourrir la relation entre la marque et le consommateur, les démarches de marketing expérientiel font largement usage du concept d'immersion (Fournier, 1998). Schématiquement, il s'agit de créer des contextes expérientiels favorisant l'immersion du consommateur, tels que les magasins, les usines, les fêtes et les sites web de marque (Caru et Cova, 2006). Les opérations de CS semblent bien s'inscrire dans ces contextes expérientiels créés par les organisations pour développer la relation du consommateur à la marque. Les opérations de CS rempliraient dès lors une double fonction s'auto-renforçant :

- Renforcement du lien entre la marque et le consommateur;

- Production et/ou sélection par le consommateur de créations authentiques.

\subsubsection{Motivations}

Le succès d'une opération de CS repose sur la participation de la foule, plus précisément sur la participation d'un nombre d'individus suffisamment important pour bénéficier de leur diversité. Il s'agit donc pour les acteurs du CS de susciter la participation de la foule. Les

\footnotetext{
${ }^{11}$ Encyclopédie du Bon Français dans l'Usage Contemporain, 1972 ; Dictionnaire Historique de la Langue Française, 1998 ; Dictionnaire du Français, 1999 ; Dictionnaire du Français, 1999 ; Dictionnaire Quillet de la Langue Française, 1975.
} 
travaux en psychologie ont montré que la motivation est un antécédent du comportement (participatif) et de la performance, la motivation étant un état psychologique dont le résultat est le comportement (Mitchell et Daniels, 2003). Ils ont également mis en lumière trois types de motivation (Deci et Ryan, 2000) : intrinsèques, extrinsèques et extrinsèques internalisées.

Les motivations typiquement intrinsèques : sont liées à la tâche. Elles apparaissent quand une activité satisfait les besoins de base d'un individu en termes de compétences, de maîtrise et d'autonomie. Il en résulte que ces activités sont intéressantes à améliorer pour elles-mêmes. Elles ne constituent pas des moyens pour aboutir à des fins. Les opérations de CS au cœur de cette recherche sont intrinsèquement motivantes, en permettant aux contributeurs d'exprimer leur créativité et au-delà de faire l'expérience du sentiment de satisfaction et d'accomplissement.

Les motivations typiquement extrinsèques : sont liées à l'environnement de la tâche. La tâche n'est pas réalisée pour elle-même mais pour ce qu'il est possible d'en retirer. C'est pourquoi les motivations extrinsèques sont caractérisées par une focalisation sur la récompense, souvent assimilable à une compensation financière. Les opérations de CS étudiées ne donnent pas toutes lieu à des rétributions financières, et quand elles sont associées à une rémunération, celles-ci sont extrêmement variables oscillant environ entre quelques centaines et 1 million d'euros. Pour les plus rémunératrices d'entre elles, les opérations de CS sont donc extrinsèquement motivantes.

Entre motivations intrinsèques et extrinsèques il existe un continuum de motivations considérées comme mixtes. Elles ne sont pas intrinsèques au départ, mais elles passent par un processus d'internalisation qui les transforme par rapport aux motivations strictement extrinsèques. Internaliser des motivations extrinsèques est notamment lié au renforcement de l'égo, au sentiment de valorisation. Cela se traduit par le fait que participer à une opération de CS peut procurer un certain statut et une visibilité au sein de la communauté des participants comme à l'extérieur d'une part et des opportunités professionnelles d'autre part. Statut/visibilité et opportunités de carrière sont donc potentiellement source de motivations importantes dans la participation aux challenges. L'internalisation de motivations extrinsèques se traduit également par l'identification à certaines actions. Par exemple, cela conduit les participants à chercher à éliminer des problèmes ou à proposer de nouvelles fonctionnalités concernant le site de CS, améliorations qu'ils perçoivent comme importantes dans leur participation immédiate au challenge. Ces actions d'amélioration sont potentiellement source de fortes motivations dans la participation aux challenges, car valorisantes auprès de la communauté des participants d'une part, et source de satisfaction voire d'accomplissement personnel d'autre part.

Afin de renforcer la participation de la foule, les acteurs du CS disposent donc d'une palette de motivations sur lesquelles ils peuvent s'appuyer par la mise en place de stimuli variés tels que l'intérêt de la tâche, la rémunération et/ou la valorisation et/ou les opportunités professionnelles pour le vainqueur du challenge. Se pose dès lors la question du ou des stimuli à privilégier pour une opération de CS donnée. Autrement dit, y-a-t-il un type de motivation plutôt qu'un autre à actionner selon les attentes attachées aux opérations de CS ?

\section{Résultats}

Ce point vise à répondre à la question générale guidant cet article : « comment une opération de CS permet-elle de créer de la valeur pour une organisation ? ». Structurés autour de deux questions intermédiaires, ces résultats établissent des liens entre différents éléments de la problématique. L'objet du premier point est d'exposer les liens entre type de foule et type d'incitation susceptible de la motiver, puis, entre type de foule et forme de valeur recherchée. Ainsi, le second point, en combinant les deux précédents liens, présentera notre réponse à la problématique de recherche. 


\subsection{Type de foule, incitations et forme de valeur recherchée}

Explicitons ici les liens entre type de foule et incitations puis forme de valeur recherchée.

\subsubsection{Comment appréhender la foule pour capter sa valeur potentielle ?}

Afin de répondre à cette première sous-question, nous nous sommes fondés sur trois éléments principaux : l'analyse du questionnaire proposé à une communauté d'internautes pratiquant le CS, les entretiens que nous avons menés et l'analyse de la littérature sur les motivations.

Si de prime abord la foule peut être assimilée à un énorme gisement de compétences anonymes, puiser efficacement dans ces dernières suppose d'établir une typologie de la masse des internautes. En effet nos investigations ont mis à jour que les individus dans la foule participent à des opérations de CS pour deux grands types de raisons : par passion et par intérêt. Sur cette base, la foule peut être scindée en deux communautés que nous dénommons : la communauté "passionnée-compétente » pour la première et la communauté « compétentepassionnée » pour la seconde, afin de rendre compte des priorités caractérisant chacune des deux communautés. Remarquons par ailleurs que ces deux communautés ne sont pas étanches, mais constituent plutôt les extrêmes d'un continuum au centre duquel se situe une communauté hybride comme l'indique la figure ci-dessous :

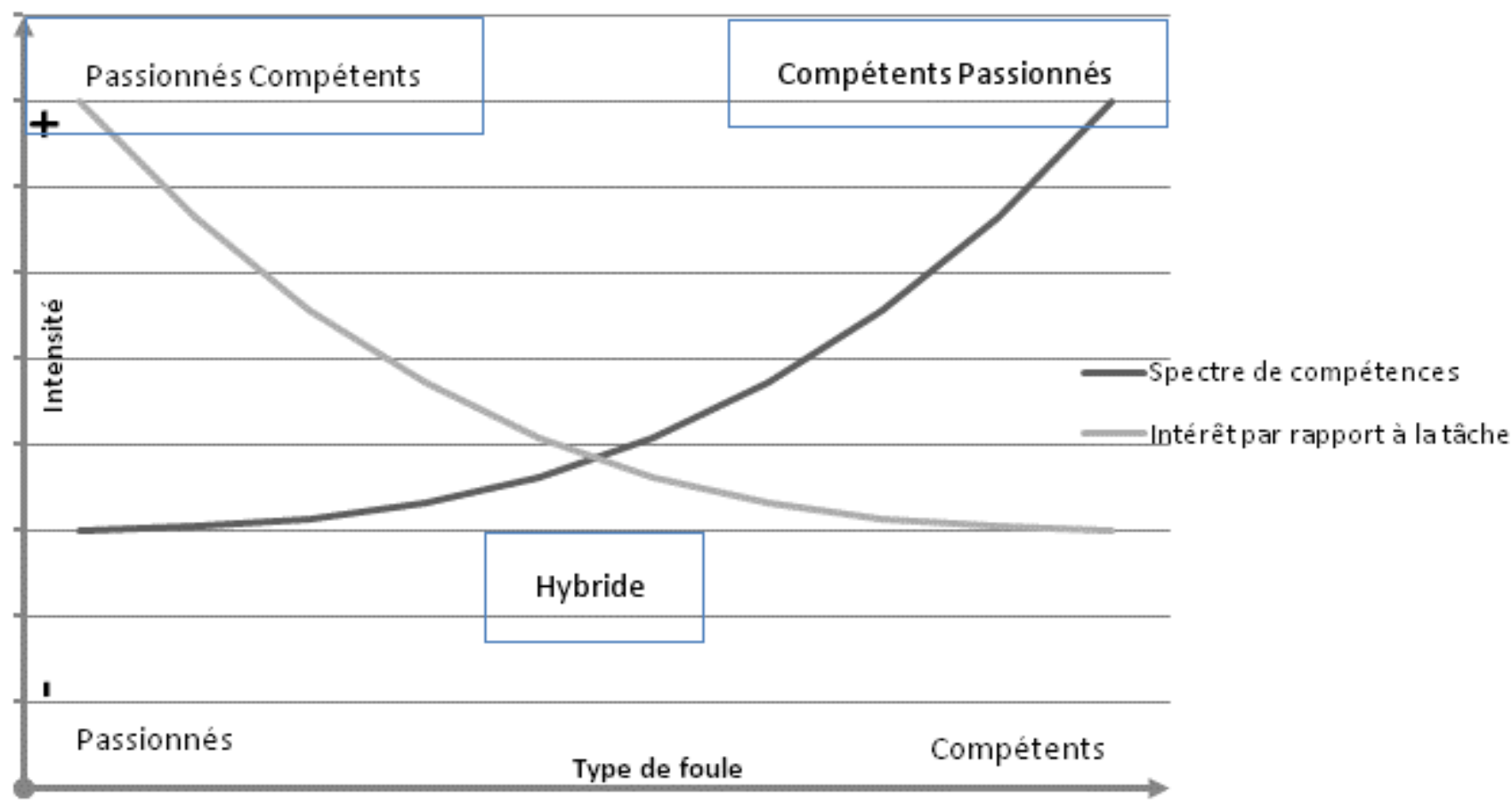

Figure 1 : Typologie de la foule

Les types de foules repérées s'avèrent fondées sur leurs motivations. C'est donc naturellement les travaux des psychologues sur le sujet qui nous ont permis d'analyser ces communautés et leurs motivations, ainsi que les incitations spécifiques à utiliser pour chacune d'entre elles.

La communauté des «passionnés - compétents» [PC]. Ce type de communauté se caractérise par le fait de porter une attention «fervente », un intérêt très important à une tâche spécifique liée fréquemment à la marque d'un bien (améliorer les produits de Dell) ou d'un service (réaliser du journalisme d'investigation au profit du journal «The Guardian »). Nous avons observé que la motivation des membres de ce type de communauté se focalise sur une volonté individuelle d'amélioration $d u$ centre d'intérêt qui les rassemble. De plus, généralement ils ne sont pas rémunérés pour leur participation. Dans ce cas, c'est la tâche en elle-même, et non pas la tâche en tant que moyen pour aboutir à des fins, qui motive avant 
tout la communauté. Ce type de foule est donc mû par des motivations intrinsèques comme l'indique le tableau suivant :

\begin{tabular}{|c|c|c|c|}
\hline $\begin{array}{l}\text { Caractéristiques de la } \\
\text { motivation intrinsèque }\end{array}$ & $\begin{array}{l}\text { Nature des } \\
\text { compétences }\end{array}$ & $\begin{array}{c}\text { Niveau et } \\
\text { spectre des } \\
\text { compétences }\end{array}$ & Exemples \\
\hline $\begin{array}{l}\text { La beauté du geste - } \\
\text { globalement, } \\
\text { désintéressée. } \\
\text { L'appartenance à une } \\
\text { communauté, un } \\
\text { réseau comme but en } \\
\text { soit. }\end{array}$ & $\begin{array}{l}\text { Focalisée sur } \\
\text { une tâche liée à } \\
\text { une marque } \\
\text { et/ou au produit } \\
\text { ou au service } \\
\text { qui lui est } \\
\text { rattaché. }\end{array}$ & $\begin{array}{l}\text { Niveau } \\
\text { variable mais } \\
\text { spectre } \\
\text { limité. }\end{array}$ & $\begin{array}{ll}\text { - } & \text { Dell } \\
\text { - } & \text { Lego } \\
\text { - } & \text { Ducati }\end{array}$ \\
\hline
\end{tabular}

Tableau 7 : caractéristiques de la communauté passionnée-compétente.

Comment alors entrer en relation avec ce type de foule et au-delà stimuler sa participation ? Les motivations intrinsèques étant liées à la tâche concernant, dans le cas du CS, une marque et/ou le produit ou service qui lui est rattaché, il convient que cette marque soit suffisamment attractive pour disposer d'une communauté, constituée essentiellement de consommateurs, de taille suffisante. L'observation par l'organisation recourant au CS, d'un comportement éthique, à l'égard de sa communauté, mais aussi concernant les valeurs «du moment» comme le développement durable ou l'écologie, s'avère également important. Il existe en effet au sein de la foule, avec une forte probabilité, des «veilleurs » sortes de gardes fous toujours prêts à dénoncer ce qui pourrait être perçu comme une forme de trahison de la communauté. Se sentant trahis, les membres de la communauté ne pourraient plus s'identifier à la marque, ce qui conduirait à une dissipation rapide de la communauté ${ }^{12}$. L'usage de techniques favorisant l'immersion du consommateur afin de renforcer les liens entre marque et consommateurs, tels que préconisé dans les démarches de marketing expérientiel est également adapté. Il s'agit en particulier d'un travail sur le site web support de l'opération de CS, et notamment sur sa convivialité.

Enfin, la participation des consommateurs passionnés sera renforcée par leur valorisation auprès de la communauté, qui consiste à montrer aux internautes que leur idée peut être réellement prise en compte et, fierté suprême, qu'elle sera alors intégrée dans les prochains produits. Le sentiment fort de «paternité partagée » du produit associé à celui d'appartenance à une communauté ou à un réseau ne peut que susciter des émules.

La communauté des «compétents - passionnés» [CP]. De manière indiscutable, les membres de ce type de communauté recherchent avant tout la satisfaction d'un intérêt matériel principalement sous forme de rémunération. Dans ce cas c'est l'environnement externe de la tâche qui motive avant tout l'internaute. La tâche constitue un moyen et non une fin, le comportement de ce type de foule étant alors régit par des motivations extrinsèques.

La communauté se constituera puis se soudera alors autour du modèle d'affaires que propose l'organisation supportant l'opération de CS. Le tableau suivant décrit les caractéristiques de ce type de communauté :

\begin{tabular}{|l|l|l|l|}
\hline $\begin{array}{c}\text { Caractéristiques de } \\
\text { la motivation } \\
\text { extrinsèque }\end{array}$ & \multicolumn{1}{|c|}{$\begin{array}{c}\text { Nature des } \\
\text { compétences }\end{array}$} & $\begin{array}{l}\text { Niveau de } \\
\text { compétences }\end{array}$ & Exemples \\
\hline Une rémunération. & $\begin{array}{l}\text { Focalisée sur les } \\
\text { demandes de celui } \\
\text { qui propose l'activité }\end{array}$ & $\begin{array}{l}\text { Variable } \\
\text { mais à à } \\
\text { tendance } \\
\text { élevé }\end{array}$ & $\begin{array}{l}\text { - InnoCentive } \\
\text { - Hypios }\end{array}$ \\
\hline
\end{tabular}

\footnotetext{
${ }^{12}$ La polémique sur l'utilisation de produits nocifs dans la marque « écolo » Ushuaïa constitue un bon exemple
} 
Quelle sont les incitations qui permettent alors d'attirer ce type de foule?

Une double idée peut être avancée ici. Premièrement, concernant le site en lui-même, il convient pour l'organisation qui gère l'opération de CS de proposer un modèle d'affaires pertinent et cohérent garantissant que l'internaute ne soit pas lésé. De ce point de vue, les questions du droit de propriété de l'idée et la rétribution du découvreur sont essentielles (Ayerbe et Chanal, 2010). Il faut éviter l'appropriation d'une idée sans rémunération de son auteur. Par ailleurs, le site supportant l'opération de CS se doit d'être respectable et crédible. La signature de contrats avec des clients reconnus par exemple contribueront à cette respectabilité. Deuxièmement, concernant les rétributions directement accordées aux «trouveurs », elles sont ici exclusivement financières. Il va s'agir de primes définies à l'avance, d'un montant élevé renforçant ainsi la crédibilité du site et clairement indiquées. En revanche, soulignons que les facteurs de réussite attachés à la précédente communauté n'apparaissent plus primordiaux. Ainsi un découvreur se sentira plus concerné par l'avantage matériel qu'il pourra retirer de la mise en service effective de son idée que par le sentiment de fierté d'en être l'auteur.

La communauté hybride. Elle comprend des personnes qui ne participent pas aux opérations de CS par pure passion pour la marque ou le produit/service associé. Mais leur participation n'est pas liée exclusivement à une rétribution financière non plus. Ce type de foule s'avère très volatile et d'un apport extrêmement variable. Les contributeurs du site Istockphoto par exemple peuvent être rattachés à cette catégorie. Leurs motivations sont certes liées au départ à une rétribution financière, mais aussi, au fur et à mesure de leur participation, leur motivation évolue vers l'appartenance à une communauté avec les opportunités qui y sont attachées en termes de visibilité notamment. Nous sommes dans ce cas face à des motivations extrinsèques internalisées.

Le tableau suivant décrit cette communauté hybride :

\begin{tabular}{|l|l|l|l|}
\hline $\begin{array}{c}\text { Caractéristiques de } \\
\text { la motivation } \\
\text { extrinsèque } \\
\text { internalisée }\end{array}$ & \multicolumn{1}{c|}{$\begin{array}{c}\text { Nature des } \\
\text { compétences }\end{array}$} & $\begin{array}{c}\text { Niveau de } \\
\text { compétences }\end{array}$ & \multicolumn{1}{|c|}{ Exemples } \\
\hline $\begin{array}{l}\text { Un statut, une } \\
\text { visibilité, } \\
\text { opportunités. des }\end{array}$ & $\begin{array}{l}\text { Focalisée sur la tâche } \\
\text { et ce que l'on peut en } \\
\text { retirer ce que l'on } \\
\text { peut retirer }\end{array}$ & Variable/Moyen & $\begin{array}{l}\text { Istockphoto } \\
\text { Les observateurs de } \\
\text { France 24 } \\
\text { Nokia Calling all } \\
\text { Innovators }\end{array}$ \\
\hline
\end{tabular}

Tableau 9 : caractéristiques de la communauté hybride

Comment inciter alors ce type de foule ? Il convient de proposer la possibilité de se faire connaître ce qui permettra par la suite, de trouver un meilleur emploi ou simplement de flatter son ego par exemple. Cependant, le caractère non tranché des motivations de cette communauté autorise également l'utilisation des stimuli propres aux deux autres communautés avec une intensité variable. Par exemple, les sommes allouées aux challenges sont généralement plus faibles.

Au terme de ce point, il s'avère que pour capter la valeur potentielle d'une foule, il convient de déterminer le type de foule répondant aux attentes attachées à l'opération de CS concernée. Ce n'est qu'ensuite que type de foule et incitations spécifiques pourront être mises en adéquation de la manière suivante : 
- Passionnés - compétents: réagissant à des motivations intrinsèques donc des incitations liées à la tâche ;

- Compétents - passionnés : réagissant à des motivations extrinsèques donc des incitations financières

- Hybride : réagissant à des motivations extrinsèques internalisées donc des incitations liées au statut et à la visibilité des individus.

Ajoutons que la mixité des motivations extrinsèques internalisées permet leur utilisation pour stimuler les deux autres types de foules.

Déterminons à présent les liens susceptibles d'exister entre type de foule et formes de valeur recherchée dans une opération de CS.

\subsubsection{Quels rapports s'établissent entre types de foule et forme de valeur ?}

Pour répondre à cette seconde sous-question, nous nous sommes fondés sur deux éléments principaux : l'observation détaillée de 63 entreprises pratiquant le CS et les entretiens que nous avons menés.

Nos analyses font nettement apparaitre des liens entre type de foule et forme de valeur. Dès lors, Pourquoi un type de foule pourrait-il plus innover qu'un autre ? La réponse est triple : compétences, diversité et mode de sélection.

Premièrement, du point de vue des compétences, innover nécessite des compétences d'un certain niveau pour pouvoir imaginer ce qu'une idée peut apporter à un produit ou service. L'authenticité quant à elle tient plus d'un sentiment exprimant la proximité d'une personne avec une marque, un produit ou un service. Ce lien de proximité s'avère indispensable dans la participation de la foule à la construction de l'identité et de l'image d'une marque. Par contre, peu de compétences semblent requises pour être authentique.

Deuxièmement, la notion de diversité, apparaît essentielle dans le processus de création innovante. En effet, créer de l'innovation requiert l'apport de multiples perspectives et connaissances, susceptibles de permettre la création d'une nouvelle idée, qui s'avère être bien souvent, la transposition d'une solution existante à un nouveau contexte. J. Surowiecki (2005) décrit fort bien l'apport de la diversité pour l'innovation : "plus important est la diversité, non pas au sens sociologique, mais plutôt conceptuel et cognitif. Vous cherchez de la diversité parmi les entrepreneurs qui viennent soumettre leurs idées, et vous finissez avec des différences significatives parmi ces idées plutôt qu'avec des variations mineures sur un même concept. » p. 63. Dans ce cadre, les membres de la communauté $\mathrm{CP}$, motivés par la rétribution financière, se caractériseront par une grande hétérogénéité des connaissances et des attitudes par rapport au produit ou au service sous-jacent à la tâche ; c'est cette diversité qui sera source d'innovation pour le produit ou le service. A l'opposé, la recherche d'authenticité conduit à établir et renforcer le lien qui unit des individus souvent consommateurs, autour d'une marque et du produit ou service qui y est associé. Il apparait dès lors une certaine homogénéité cognitive au sein des membres de la communauté PC, qui s'agrègent via des motivations intrinsèques liés à la marque et au produit ou au service associé.

Troisièmement, le mode de sélection s'avère également déterminant. Dans le cadre de l'authenticité, il s'agit de chercher la tendance lourde, majoritaire qu'exprime la foule au regard d'une marque et du produit ou service associé. Il est extrêmement ardu pour une personne ou un petit groupe de déterminer cette tendance. Cela s'explique par le nécessaire traitement d'un très grand nombre d'avis, d'idées ou d'opinions, de qualité souvent moyenne voire médiocre. Aussi, il apparait plus efficace de laisser la foule opérer cette sélection pour faire émerger les idées qui la représente le mieux. C'est ainsi que le mécanisme de vote s'applique très bien à ce processus, que l'on pourrait qualifier de démocratique. De plus comme le précise Howe dans son ouvrage : «la foule a toujours raison » (Howe, 2009: 287). Concernant l'innovation, il s'agit au contraire de repérer la «pépite» dans le tamis du 
chercheur d'or. Là, seul l'œil averti d'un expert ou d'un groupe d'experts pourra comprendre la portée d'une idée pour un problème et juger de sa faisabilité. Ici, la sélection s'opère par l'entreprise qui recourt au CS ou par une équipe mêlant cette entreprise et le prestataire de service gérant l'opération, mais ce n'est pas la foule qui sélectionne. C'est donc bien le référentiel minimum, c'est-à-dire l'organisation, qui est utilisé pour juger de l'innovation apportée à un produit, un service ou un procédé.

Le tableau ci-dessous résume notre propos et illustre les liens entre type de foule et forme de valeur recherchée.

\begin{tabular}{|c|c|c|c|}
\hline \multirow{4}{*}{ 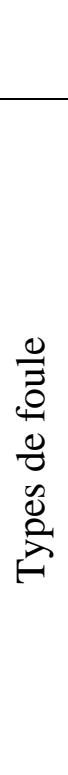 } & \multirow[b]{3}{*}{$\begin{array}{l}\text { compétents - } \\
\text { passionnés }\end{array}$} & \multicolumn{2}{|c|}{ Forme de valeur recherchée } \\
\hline & & Authenticité & Innovation \\
\hline & & $\begin{array}{l}\text { Difficile à définir, car les } \\
\text { solutions proposées sont } \\
\text { liées à l'avantage attendu (la } \\
\text { rétribution financière) et } \\
\text { non à la marque, au produit } \\
\text { ou au service. }\end{array}$ & $\begin{array}{l}\text { Création de valeur par la } \\
\text { diversité conceptuelle et } \\
\text { cognitive. } \\
\text { Compétences et distance } \\
\text { facilitent la création } \\
\text { d'innovations. } \\
\text { L'entreprise sélectionne }\end{array}$ \\
\hline & $\begin{array}{l}\text { passionnés - } \\
\text { compétents }\end{array}$ & $\begin{array}{l}\text { Création de valeur par la } \\
\text { proximité entre foule, la } \\
\text { marque et le produit ou le } \\
\text { service. } \\
\text { Emergence d'un sentiment } \\
\text { dominant. } \\
\text { La foule sélectionne }\end{array}$ & $\begin{array}{l}\text { La faible } \quad \begin{array}{l}\text { diversité } \\
\text { compétences }\end{array} \text { réduit } \\
\text { probabilité d'occurrence d'une } \\
\text { innovation. }\end{array}$ \\
\hline
\end{tabular}

Tableau 10 : liens entre type de foule et forme de valeur recherchée

Examinons enfin le cas de la foule hybride. Ce type de foule est mû par des motivations extrinsèques internalisées et réagit en particulier aux incitations qui augmentent la visibilité des membres au sein de la communauté et qui offrent des opportunités professionnelles. A ce stade de nos investigations, nous estimons que ce type de foule peut proposer des améliorations à des produits existants. Cependant, l'éloignement de la foule et du produit peut conduire à ce que ces améliorations ne soient pas partagées par la communauté des consommateurs du produit. Il ne s'agit donc pas ici d'innovations radicales, ni de créations authentiques.

Les deux modes de sélection des idées ont été observés; le choix de l'un ou de l'autre étant lié au niveau de contrôle que souhaite conserver l'entreprise sur ce qui va émerger. Par exemple, dans le cas des observateurs de France 24, la sélection des reportages amateurs est effectuée par la chaîne d'information. Dans le cas du site Istockphoto, le classement des meilleures photos s'opère par les votes des membres du site. L'observation attentive de ces deux cas met en lumière que l'on y trouve des créations moyennement authentiques (car filtrées par la chaîne dans le cas des reportages d'actualité ou artificiellement embellies par leurs propres auteurs afin rendre leurs photos plus vendables, ceci au détriment de leur véritable sensibilité source d'authenticité) et des innovations plutôt incrémentales (ces 2 sites n’ont ni révolutionné les reportages d'actualité, ni le monde de la photo).

\subsection{Vers un Business Model adapté au crowdsourcing}

En réponse à notre interrogation initiale : «Comment une opération de CS permet-elle de créer de la valeur pour une organisation ? », nous proposons les bases d'un Business Model adapté aux entreprises : 
- gérant elles-mêmes leurs opérations de CS. Elles externalisent certaines activités auprès de la foule, mais elles le font sur leur propre site web ou sur un site dédié qu'elles ont crée à cet effet ;

- d'intermédiation de CS, autrement dit des entreprises dont le CS constitue le cœur de métier en gérant des opérations de CS pour le compte d'organisations clientes.

Comme le proposent Osterwalder et al., (2009), un BM peut être détaillé à partir d'un canevas comportant neuf blocs. Nous nous focaliserons ici sur trois blocs en particulier: l'offre de valeur, les ressources clés et les activités clés. L'offre de valeur représente la forme de valeur recherchée par le client et proposée par l'entreprise. Les ressources clés sont les éléments sur lesquels une organisation s'appuie pour nourrir ses activités clés et ainsi créer de la valeur. Les activités clés représentent les opérations essentielles qu'effectuent l'organisation et qui mènent à la création de valeur. Dans notre cas, l'offre de valeur s'appuie sur l'explicitation, par l'entreprise qui souhaite externaliser une de ses activités auprès de la foule, de ses attentes en termes d'authenticité ou d'innovation concernant l'opération de CS. Les ressources clés sont constituées par la foule et plus précisément par le type de foule qui sera ciblé. Les activités clés sont constituées par la génération et/ou la sélection des contenus opérée par la foule. Pour reprendre la taxinomie proposée par Osterwalder et al., pp. 138-139, l'épicentre de ce BM a pour origine l'offre de valeur.

Le processus permettant de définir un BM cohérent pour une opération de CS donnée s'appuie sur les réponses aux deux sous-questions de recherche exposées précédemment. Ce processus se déroule concrètement de la manière suivante :

1. Déterminer la forme de valeur que l'entreprise souhaite ajouter à son activité : innovation ou authenticité.

2. Selon la forme de valeur recherchée, un certain type de foule doit être visé. Une foule ne participant pas spontanément, il convient également de choisir des incitations répondant aux motivations animant le type de foule recherché. Parmi la palette d'incitations disponibles, il conviendra de les déterminer finement et de mettre en place celles qui sont adaptées (de la rémunération à la valorisation de l'idée d'un individu en passant par la mise en place de fonctionnalités assurant la visibilité et au-delà des perspectives d'embauche par exemple).

3. Enfin, selon la forme de valeur recherchée et le type de foule visé, il est nécessaire de définir le rôle dévolu à la foule, en termes de génération et/ou de sélection de contenu. Dans cet article, nous nous sommes focalisés sur une génération de contenu opérée par la foule, associée à une sélection du contenu prise en charge, soit par la foule, soit par l'entreprise selon la forme de valeur recherchée. Le cas dans lequel l'organisation génère elle-même du contenu et délègue uniquement à la foule l'opération de sélection, n'a pas été étudié. Cette approche constitue d'ailleurs une piste de recherche qu'il conviendra d'explorer ultérieurement.

La figure suivante résume le processus permettant de créer de la valeur à travers une opération de CS : 


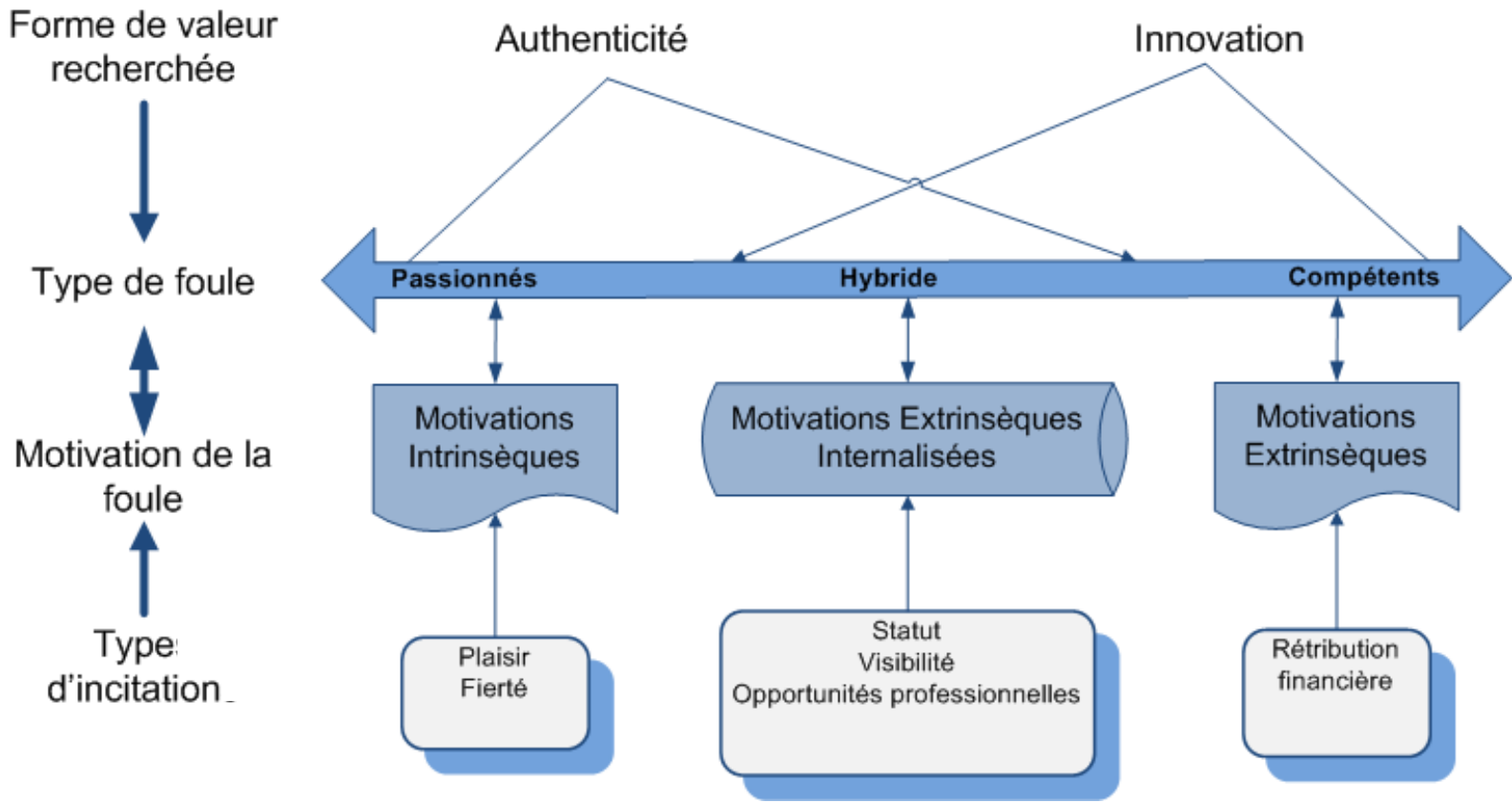

Figure 2 : processus de création de valeur par une opération de crowdsourcing

Le processus permettant à une organisation de créer de la valeur, en termes d'innovation ou d'authenticité, dans le cadre d'une opération de CS a été exposé. Présentons à présent trois exemples emblématiques de CS, de manière à illustrer ce processus en observant, les incitations mises en œuvre dans le cadre de ces opérations de CS afin d'en déduire, le type de motivation et donc le type de foule attiré, et au-delà d'estimer la forme de valeur créée pour l'entreprise.

\section{Conclusion autour d'exemples emblématiques}

En un peu moins de quatre ans, le nombre de sites se déclarant faire appel au concept de crowdsourcing n'a cessé d'augmenter. Actuellement, le site Openinnovators notamment, renferme près d'une centaine d'exemples. Les trois illustrations choisies ici nous paraissent remarquables car à la fois comparables (leur objet réside dans la génération d'idées) et pourtant différentes (nous le verrons en synthèse), mais aussi représentatives de l'ensemble des autres sites de crowdsourcing que nous avons observés, et enfin permettant de discuter et d'expliciter les résultats précédemment présentés.

Chaque cas sera d'abord brièvement présenté, puis nous spécifierons le type d'incitation proposé, la forme de valeur recherché et ainsi le type de communauté y participant.

\subsection{Améliorer ou innover? Le cas de Dell IdeaStorm}

Lancé par Dell en février 2007, IdeaStorm vise à l'externalisation d'une partie des activités de R\&D de Dell. Pour ce faire Dell indique vouloir profiter de ses millions de clients répartis dans plus de 100 pays. Le principe paraît simple : une fois enregistré, un individu propose une idée afin d'améliorer le fonctionnement d'un produit ou d'un service de Dell. Cette idée est ensuite affichée sur le site et deux boutons permettent aux internautes soit de la promouvoir soit de l'éliminer ${ }^{13}$. Au $1^{\text {er }}$ Août 2010, la communauté des internautes a proposé 14427 idées ; 727628 votes et 89812 commentaires ont concerné ces idées. Plus de 400 idées ont été retenues et incorporées dans les solutions Dell. Notons enfin que Dell a intégré la notion de

\footnotetext{
${ }^{13}$ Ce processus de vote remarquablement efficace n'a pas été développé par Dell mais constitue un produit à part entière proposé par la société Salesforce qui offre des services de CRM et de cloud computing.
} 
crowdsourcing dans son organisation puisqu'a été créé la fonction de Vice-président en charge des communautés et des échanges ${ }^{14}$.

Voyons à présent les incitations mises en œuvre pour stimuler la participation des internautes. Les auteurs ne sont pas rémunérés pour leurs «trouvailles», identifiés sous leur nom de connexion ils ne sont pas individuellement valorisés. Enfin, ils ne sont visibles que très ponctuellement ${ }^{15}$, lorsque la liste récapitulant les idées retenues et le nom de connexion de leurs auteurs est publiée dans une sous-partie du site général de Dell intitulé Direct2Dell.

L'exemple d'IdeaStorm apparaît symbolique pour au moins deux raisons. En tant que leader dans la vente de matériel informatique, le fait que Dell recourt à ce site témoigne d'un mouvement de grande ampleur. De plus, Dell a toujours été reconnu comme une entreprise ne menant pas d'actions de R\&D. Ainsi, avec IdeaStorm, Dell se lance dans la R\&D sans l'avoir jamais intégrée, autrement dit en passant directement par une externalisation ouverte. Mais quelle forme de valeur crée Dell via ce site ? Certains estiment que Dell recherche des créations innovantes (Di Gangi et Wasko, 2009), nous n'en sommes pas si certain. En effet, à y regarder de près, les idées intégrées par Dell concernent des améliorations assez classiques du matériel existant (par exemple ajouter de la RAM à un type de matériel) ou bien consistent à ajouter de nouvelles fonctionnalités aux services offerts (notamment lors de la commande). Aucune innovation radicale n'est présentée. De notre point de vue, ceci est assez logique. En effet, avec le type d'incitations mis en place, Dell va principalement attirer des "passionnés » qui proposeront des idées visant à améliorer les réalisations de Dell (par définition ils ont une ancienne version du matériel qu'ils proposent d'améliorer). Ainsi Ideastorm favorise la création d'authenticité et permet à Dell d'offrir des produits proches des préoccupations de ses utilisateurs... Point d'innovation radicale ici... La récente initiative de créer des «storm sessions ${ }^{16}$ portant sur des thèmes larges pourrait constituer une ré-orientation vers la création d'innovations, encore faudra-t-il mettre en place des incitations susceptibles d'attirer une foule aux compétences diversifiées. A ce jour, rien n'augure ce changement d'incitations. Nous estimons dès lors que Dell n'a pas pour objectif, du moins à court terme, de rechercher des innovations radicales à travers ses opérations de crowdsourcing.

\subsection{Le carrefour des innovations : InnoCentive}

Fondée en 2001, InnoCentive vise à mettre en relation des organisations en recherche d'idées innovantes avec un vaste réseau de chercheurs. Les organisations peuvent être de nature différente, comme par exemple, des entreprises publiques ou privées, des centres académiques de recherche ou encore des ONG. InnoCentive se propose de garantir la fiabilité et la légitimité des entreprises qui affichent des demandes et proposent une rémunération pour « les chercheurs qui trouvent». Une opération de crowdsourcing via InnoCentive obéit au processus suivant : les problèmes sont mis en ligne sur le site avec un résumé de la demande, une date limite de réponse et le montant de la récompense attribuée à la meilleure solution (entre 5000 et $1250000 \$ \$^{17}$ ). Pour afficher une demande, l'entreprise demandeuse paie un acompte à InnoCentive. Pour avoir plus de précisions sur la demande, les internautes doivent être inscrits comme «découvreurs de solution». Les scientifiques du monde entier sont

\footnotetext{
${ }^{14}$ Le poste de «VP of communities and conversations » a été crée (dirigé par Bob Pearson jusqu'en fin 2008) et depuis, cette fonction s'appelle «VP of Social Media and Community (dirigée par Manish Mehta).

${ }^{15}$ Par exemple, au 15 juillet 2010, le dernier tableau récapitulatif des idées retenues dataient du 22 janvier 2010.

${ }^{16}$ Depuis fin décembre 2009, Dell organise des sessions appelées «storm sessions » qui ont pour objectif de générer des idées. Ces sessions sont organisées autour de problématiques managériales exprimées par les responsables de Dell (Green IT par exemple). Dans ce cadre, la communauté poste des idées et vote sur ces idées. Des sessions internes sont ensuite organisées, au sein desquelles les idées des internautes sont discutées et sélectionnées puis annoncées à la communauté.

${ }^{17}$ Un challenge d'un tel montant est actuellement en cours pour proposer une innovation concernant les protéines actives dans les insecticides.
} 
éligibles et peuvent aisément s'inscrire. Une fois le problème résolu, InnoCentive prend en charge le transfert des droits de propriété intellectuelle ainsi que le paiement. Actuellement plus de 150 problèmes sont en cours de proposition et une foule de plus de 200000 scientifiques est enregistrée comme «apporteurs de solutions » (solvers). Enfin près de 700 challenges ont été remportés. Les scientifiques inscrits proviennent de plus d'une centaine de pays et plus de la moitié sont localisés hors des USA. Le spectre des compétences impliquées apparaît donc vaste.

Ajoutons que le site est géré par une équipe clairement identifiée de managers ayant déjà acquis une expérience reconnue et que les aspects légaux et contractuels sont parfaitement formalisés.

Selon notre grille de lecture, Innocentive constitue une plateforme sérieuse permettant de créer de l'innovation. Les incitations proposées attirent incontestablement une foule de compétents - passionnés aux motivations extrinsèques. Le modèle d'affaires nous apparaît cohérent, d'ailleurs Innocentive va prochainement fêter ses dix ans d'existence et de nombreux sites clones ont vu le jour au cours de cette décénnie.

\subsection{La voie médiane : Nokia Calling All Innovators}

Depuis 2 ans, nous suivons de près ce site de CS, qui illustre bien le recours à une communauté hybride. Créé par Dean Kamen, l'inventeur du célèbre Segway, ce site a pour but de rattraper le retard de Nokia sur Apple en termes d'offres applicatives. Nokia Calling All Innovators [NCAI] a ceci de passionnant qu'il propose depuis peu un nouveau type de challenge. Au départ, NCAI visait à la création d'applications et de services pour Nokia et proposait des incitations mêlant rétributions financières (de 10000 à 30000 \$) et une exposition d'une durée variable sur le site de vente d'applications de Nokia pour le découvreur. L'entreprise recherchait à la fois de l'innovation et de l'authenticité et usait pour cela d'un système d'incitations mixte lié aux différents type de motivation. L'année $2010^{18} \mathrm{a}$ vu le lancement d'un nouveau défi intitulé : «Growth Economy Venture Challenge ». Il s'agit de proposer un produit ou un service lié au domaine de la mobilité et qui améliorera la vie des personnes situés dans des pays en développement. Le prix sera une aide pour le financement de cette solution d'un montant de 1 million de \$. Nous sommes ici face à une valeur hybride. En effet, d'une part, Nokia recherche de l'authenticité pour créer une proximité entre sa marque et une solution répondant à des besoins éthiques. D'autre part, Nokia est en quête d'innovation radicale pour essayer de transformer l'existence de millions de personnes. L'incitation proposée renferme en elle cette hybridité. En effet, d'une part, le montant est élevé et peut engendrer des motivations extrinsèques, mais d'autre part, il s'agit du financement d'un projet et rien ne dit que le découvreur gagnera de l'argent avec ce prix. Il pourrait intégralement le dépenser pour financer la mise en œuvre de son idée, ce qui correspondrait à une motivation plutôt intrinsèque.

Au terme de trois années d'exploration et de réflexion autour du concept de crowdsourcing, à travers une approche de la valeur adaptée au crowdsourcing sélectif, nous avons proposé un réseau de relations associant type de foule, type de motivation et d'incitation avec la forme de valeur recherchée. S'agissant d'une étude exploratoire, il convient désormais de tester ces résultats par des études spécifiques à chaque relation.

L'identification de deux formes de valeur distinctes recherchées dans les opérations de CS créations innovantes et créations authentiques- constitue l'un des apports majeur de cette recherche. En effet, en dépassant les approches exclusivement centrées sur la recherche

\footnotetext{
${ }^{18}$ Période pour soumettre le projet : janvier - juillet 2010. Décision finale le 15 septembre 2010.
} 
d'innovation, cette distinction permet de rendre compte de la diversité des pratiques observées, elle constitue dès lors le point de départ du Business Model proposé.

Enfin, si ce travail, en montrant comment le CS créé de la valeur pour une organisation, véhicule une vision plutôt optimiste de cette modalité d'externalisation, soulignons qu'il ne constitue qu'une solution parmi d'autres à la disposition des organisations. C'est ce qu'illustre parfaitement le cas des 908 idées proposées par la communauté d'Innocentive ${ }^{19}$ pour remédier à la fuite de pétrole dans le golfe du Mexique, toutes rejetées par BP qui a préféré suivre les solutions préconisées par ses propres ingénieurs, dont la supériorité en terme d'efficience est loin d'être démontrée !

\section{Webographie}

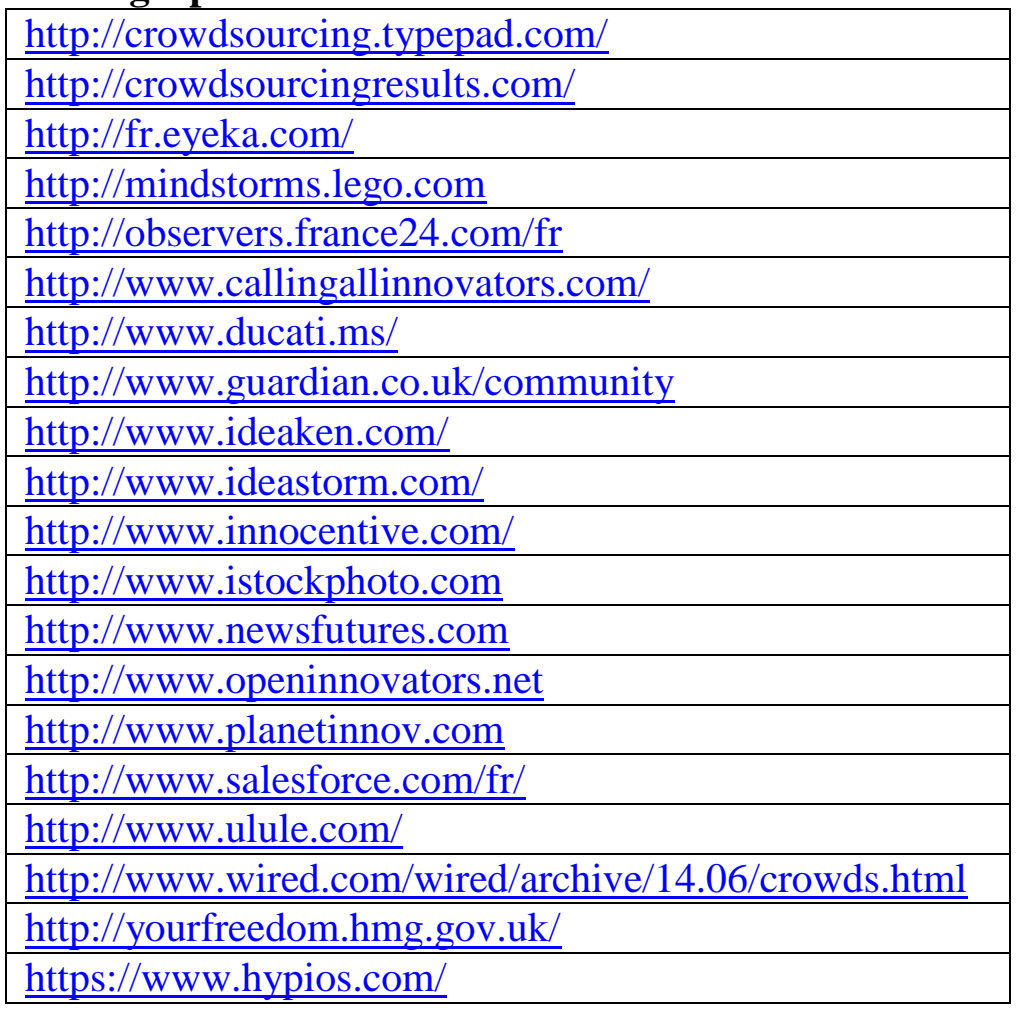

\section{Bibliographie}

Aguila-Obra, A. R. d., Padilla-Meléndez, A. \& Serarols-Tarrés, C. (2007), Value creation and new intermediaries on Internet. An exploratory analysis of the online news industry and the web content aggregators. International Journal of Information Management, Vol. $27 \mathrm{n}^{\circ} 3,187-99$.

Amit, R. \& Zott, C. (2001), Value Creation in e-Business. Strategic Management Journal, Vol. $22 \mathrm{n}^{\circ}, 493-520$.

Ayerbe, C. (2006), Innovation technologique et organisationnelle au sein de P.M.E. innovantes : complémentarité des processus, analyse comparative des mécanismes de diffusion. Revue Internationale PME, Vol. $19 \mathrm{n}^{\circ}$ 1, 9-34.

Ayerbe, C. \& Chanal, V. (2010), Quel management des droits de propriété intellectuelle dans les business models ouverts : une lecture critique des travaux de Chesbrough. Revue Française de Gestion, Vol. A Paraître ${ }^{\circ}$.

Blanchet, A. \& Gotman, A. (1992), L'enquête et ses méthodes : l'entretien. Paris: Nathan.

Caru, A. \& Cova, B. (2006), Expériences de marque : comment favoriser l'immersion du consommateur ? Décision Marketing, Vol. $41 \mathrm{n}^{\circ}, 43-52$.

\footnotetext{
${ }^{19}$ Pour plus d'informations, faire une recherche sur «BP to Innocentive: Sorry, We Don't Want Your 908 Ideas for Saving the Gulf »
} 
Chesbrough, H. (2006a), New Puzzles and New Findings. Dans H. Chesbrough, W. Vanhaverbeke \& J. West (Eds.) Open Innovation: Researching a New Paradigm: 1534. Oxford University Press.

Chesbrough, H. (2006b), Open Business Model : How to thrive in the new innovation landscape. Harvard Business School Press.

Chesbrough, H. W. \& Appleyard, M. M. (2007), Open Innovation and Strategy. California Management Review, Vol. $50 \mathrm{n}^{\circ}$ 1, 57-76.

Cova, V. \& Cova, B. (2001), Alternatives Marketing. Paris: Dunod.

Crossan, M. \& Apaydin, M. (2010), A Multi-Dimensional Framework of Organizational Innovation: A Systematic Review of the Literature. Journal of Management Studies, Vol. $47 \mathrm{n}^{\circ} 6,1154-91$.

Deci, E. L. \& Ryan, R. M. (2000), The "what" and "why" of goals pursuits: human needs and the self-determination. Psychology Inquiry, Vol. $11 \mathrm{n}^{\circ}$ 4, 227-68.

Di Gangi, P. M. \& Wasko, M. (2009), Steal my idea ! Organizational adoption of user innovations from a user innovation community:A case study of Dell IdeaStorm. Decision Support Systems, Vol. 48 n $^{\circ}$ 1, 303-12.

Fournier, S. (1998), Consumers and their brands: developing relationship theory in consumer research. Journal of Consumer Research, Vol. $24 n^{\circ}, 343-73$.

Gaspoz, C. (2010), Prediction Markets Supporting Technology Assessment. HEC Lausanne. Lausanne: Université de Lausanne.

Girotra, K., Terwiesch, C. \& Ulrich, K. (2010), Idea Generation and the Quality of the Best Idea. Management Science, Vol. $56 \mathrm{n}^{\circ}$ 4, 591-605.

Gobe, M. (2001), Emotional branding: the new paradigm for connecting brands to people. New York: Allworth Press.

Gosain, S. (2003), Looking through a Window on Open Source Culture: Lessons for Community Infrastructure Design. Systèmes d'Information et Management, Vol. $8 \mathrm{n}^{\circ}$ $1,11-42$.

Guittard, C. \& Schenk, E. (2010), Le Crowdsourcing : Une typologie des pratiques d'externalisation vers la foule. Paper presented at XIXème conférence de l'AIMS.

Howe, J. (2006), The Rise of Crowdsourcing. Wired, Vol. $14 \mathrm{n}^{\circ}$ 6, 134-45.

Howe, J. (2009), Crowdsourcing: Why the Power of the Crowd is Driving the Future of Business. New York: Three rivers Press.

Johannessen, J.-A. \& Olsen, B. (2010), The future of value creation and innovations: Aspects of a theory of value creation and innovation in a global knowledge economy. International Journal of Information Management, Vol. In Press, Corrected Proof $\mathrm{n}^{\circ}$.

Kim, C., Oh, E., Shin, N. \& Chae, M. (2010), An empirical investigation of factors affecting ubiquitous computing use and U-business value. International Journal of Information Management, Vol. In Press, Corrected Proof $\mathrm{n}^{\circ}$.

Klein, H. K. \& Myers, M. D. (1999), A Set of Principles for Conducting and Evaluating Interpretive Field Studies in Information System. Management Information System Quaterly, Vol. $23 \mathrm{n}^{\circ} 1,67-94$.

Kolar, T. \& Zabkar, V. (2010), A consumer-based model of authenticity: An oxymoron or the foundation of cultural heritage marketing? Tourism Management, Vol. $31 \mathrm{n}^{\circ}$ 5, 65264.

Le Bon, G. (2003), Psychologie des foules. Paris: PUF.

Lebraty, J. F. (2009), Externalisation ouverte et pérennité : une nouvelle étape de la vie des organisations. Revue Française de Gestion, Vol. n ${ }^{\circ}$ 192, 151-65.

Lefebvre, P., Roos, P. \& Sardas, J. C. (2004), Les théories des Communautés de Pratique à l'épreuve : Conditions d'émergence et Organisation des communautés. Systèmes d'Information et Management, Vol. $9 \mathrm{n}^{\circ}$ 1, 25-48. 
Lepak, D. P., Smith, K. G. \& Taylor, M. S. (2007), Value Creation And Value Capture: A Multilevel Perspective. Academy of Management Review, Vol. $32 \mathrm{n}^{\circ}$ 1, 180-94.

MacIntosh, A. \& Prentice, R. C. (1999), Affirming authenticity. Consulting cultural heritage. Annals of Tourism Research, Vol. $26 \mathrm{n}^{\circ}$ 3, 589-612.

Mitchell, T. R. \& Daniels, D. (2003), Motivation. Dans I. B. Weiner (Ed.) Handbook of Psychology. Industrial and Organizational Psychology: 225-54. New York: Wiley.

Modell, S. (2005), Triangulation between case study and survey methods in management accounting research: An assessment of validity implications. Management Accounting Research, Vol. $16 \mathrm{n}^{\circ}$ 2, 231-54.

Moe, N. B., Dingsoyr, T. \& Dyba, T. (2010), A teamwork model for understanding an agile team: A case study of a Scrum project. Information and Software Technology, Vol. 52 $\mathrm{n}^{\circ} 5,480-91$.

Möller, K. \& Rajala, A. (2007), Rise of strategic nets - New modes of value creation. Industrial Marketing Management, Vol. $36 \mathrm{n}^{\circ}$ 7, 895-908.

Mors, M. (2010), Innovation in a global consulting firm: when the problem is too much diversity. Strategic Management Journal, Vol. $31 \mathrm{n}^{\circ}$ 8, 841-72.

Osterwalder, A., Pigneur, Y. \& Smith, A. (2009), Business Model Generation. Self Published.

Priem, R. L. (2007), A Consumer Perspective On Value Creation. Academy of Management Review, Vol. $32 \mathrm{n}^{\circ} 1,219-35$.

Surowiecki, J. (2005), La sagesse des foules. Paris: JC Lattès.

Thiétard, R. A. (1999), Méthodes de recherche en management. Paris: Dunod.

Welch, I. (2000), Herding among security analysts. Journal of Financial Economics, Vol. 58 $\mathrm{n}^{\circ} 3,369-96$. 